\title{
Development process of new bumper beam for passenger car: A review
}

\author{
M.M. Davoodi a,b,, S.M. Sapuan b, A. Aidy c, N.A. Abu Osman a, A.A. Oshkour a, W.A.B. Wan Abas a \\ a Department of Biomedical Engineering, Faculty of Engineering, University of Malaya, UM, 50603 Kuala Lumpur, Malaysia \\ b Department of Mechanical and Manufacturing Engineering, University Putra Malaysia, UPM, Serdang, 43400 Selangor, Malaysia \\ c Department of Mechanical Engineering, National Defence University of Malaysia, UPNM, 57000 Kuala Lumpur, Malaysia
}

Introduction

Design is the preliminary stage of product development and analysis. The embodiment stage of the design process fairly predicts the failure(s), if any, before mass production. Passenger vehicles make up over $90 \%$ of the fleet of registered vehicles. In 2009 it was estimated that 9,640,000 vehicles were involved in police-reported crashes, $95 \%(9,161,000)$ of which were passenger vehicles. Furthermore, there, 45,435 vehicles of these were involved in fatal crashes and eighty percent of which $(36,252)$ were passenger vehicles. More than 23,000 passenger vehicle travelers lost their lives in traffic crashes in 2009 and an estimated 1.97 million persons were injured [1]. Therefore, vehicle safety requirements forced by Governments and insurance companies increase frequently [2]. In most of the accidents, the bumper system is the first vehicle part that receives the collision and which may to some extent protect the car body and passengers. This system comprises three main parts: fascia, energy absorber, and bumper beam [3]. The fascia is a non-structural aesthetics component that reduces the aerodynamic drag force while the energy absorber dissipates part of the kinetic energy during collision. The bumper beam is a structural component which absorbs the low-impact energy by bending resistance and dissipates the high-impact energy by collision [4].

There are some investigations of new material development, property improvement, and FEA of bumper beam structures by researchers and car manufacturers. These parties are mainly interested in substituting the conventional material with lighter and stronger material [5]. Renault used SMC in a passenger car bumper in 1972 instead of steel [6] and General Motors (GMs), used the sheet molding compound (SMC) beam in Pontiac Bonneville Cadillac Seville and Cadillac Eldorado instead of steel which was used in previous models [7]. Cheon et al. [8] found that the polymer composite 
bumper beam offers $30 \%$ less weight than steel without

scarifying the bumper beam's bending strength. Wakeman et al.

[9] found that holding time pressure is the most effective parameter

among five processing parameters in microstructure and macrostructure

properties of glass mat thermoplastic (GMT) in a

bumper beam. Peterson et al. [10] from Azdel company developed

the GMT with a high surface finish for aesthetic components. Raghavendran

and Haque [11] also developed a lightweight GMT composite

containing long-chopped fiber strands to be used in headliner and other automotive interior applications. Suddin et al.

[12] used the weight analysis method to select fascia for a desired

vehicle. He used the knowledge-based system (KBS) approach to

select the material for bumper beam development [13]. Sapuan

et al. [3] studied the conceptual design and material selection for development of a polymeric-based composite automotive bumper system. Hosseinzadeh et al. [14] studied the shape, material, and impact conditions of the bumper beam and compared the results with conventional metals like steel and aluminium. He found that GMT can replace SMC as a recyclable material. Kokkula et al. [15] experimentally studied bumper beam performance at $40 \%$ offset impact crashworthiness and concluded that materials with moderate strain-hardening properties are preferable over the higher strain-hardening materials for his studied system. Hambali et al. [16] studied employed the analytical hierarchy process (AHP) in concept selection of bumper beam during the conceptual design stage of product development. Marzbanrad et al. [17] studied bumper beam crashworthiness improvement by analyzing bumper beam material, thickness, and shape as well as impact condition parameters. He found that a modified SMC bumper beam is preferable to the ribbed GMT bumper beam as the former has the potential to minimize the bumper beam deflection, impact force, and stress distribution and to maximize the elastic strain energy while exhibiting almost the same energy absorption of the unribbed SMC bumper beam. Park et al. [18] developed an optimized bumper beam cross section that satisfies both the safety requirements for a front rigid-wall impact and lower leg injuries in a pedestrian impact test. Most of the abovementioned research emphasizes on material and concept selection for, and numerical analysis of, bumper beam. However, no articles regarding procedure(s) for new bumper beam development could be found in the open literature.

This study therefore focuses on the process of bumper beam development and summarizes the method of design and analysis of the new bumper beam in new vehicle development based on the previous research and the authors' personal experiences. In consequence, this article helps the designer to follow the right procedure for bumper beam development. It emphasizes on the parameters that have to be considered in the design of bumper beams and illustrates the procedure for FE analysis the bumper 
system.

2. Bumper system

\subsection{Bumper system definition}

A bumper system is a set of components in the front and rear parts of the vehicle designed for damping the kinetic energy without any damage to the vehicle in low-speed impact and for energy dissipation in high-speed impact conditions besides serving aesthetic and aerodynamic purposes [19,20]. A bumper system mainly comprises three components: fascia, energy absorber, and beam [3].

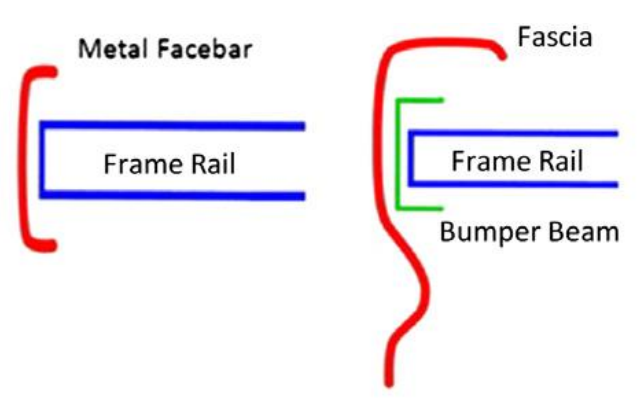

(1)

(2)

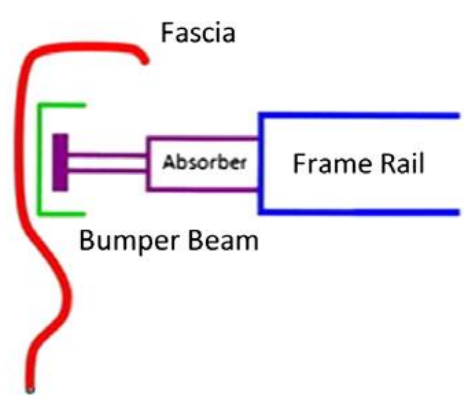

(3)

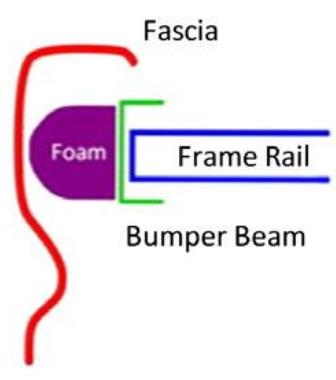

(4)

Fig. 1. Common bumper systems.

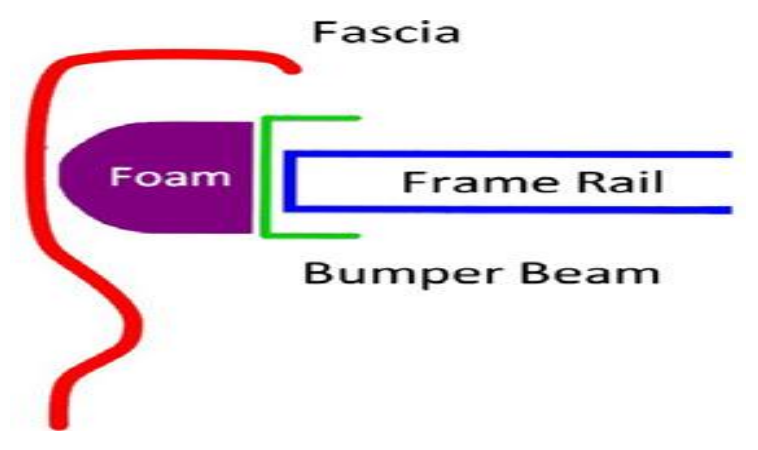

Fig. 2. Pedestrian, low impact and crash impact system.

The bumper system has changed over the last three decades due to new government safety regulations and styling concepts. The ability to maintain the vehicle intact at high-speed impact conditions and to damp the kinetic energy are the most important factors in bumper system selection besides its weight, manufacturability, cost, reparability, and formability of materials [21] (Fig. 1).

The American Iron and Steel Institute [22] offered four proposals for bumper systems: (1) metal face bar, (2) plastic fascia and reinforcing beam, (3) plastic fascia reinforcing beam and mechanical energy absorbers, and (4) plastic fascia reinforcing beam and foam, or honeycomb, energy absorbers. According to the new regulation, 
the pedestrian leg impact test was due to be enacted and implemented starting from 2010. Some research has been carried out to offer methods for complying with the pedestrian impact test. The energy absorption density in the low-impact test approximately doubled in comparison with the pedestrian impact [23].

Choi et al. [24] came up with the concept of locating the energy absorber between the bumper fascia and the reinforcement beam to absorb the impact energy when the second energy absorber is subjected to an impact greater than its critical elastic force. Therefore, this concept (to be referred to hereafter as concept number 5 or concept No. 5) can be added to the four bumper system components which the American Iron and Steel Institute (AISI) offered in 2003 (Fig. 2) which is a schematic view of a concept No. 5 system modified from AISI for car bumper system. In this method, two types of energy absorbers are considered: firstly, a low stiffener absorber, which is called the reversible absorber, is designed for protection against low and pedestrian impact; and secondly, the irreversible energy absorber, which comprises the beam and the crushable energy absorber and is usually located at the back of the beam and attached to the main face bar.

\subsection{Bumper beam definition and function}

The bumper beam is the backbone of the energy-absorption mechanism of the bumper system [8]. It is usually located in the front, and sometimes in the rear, sides of the vehicles. However

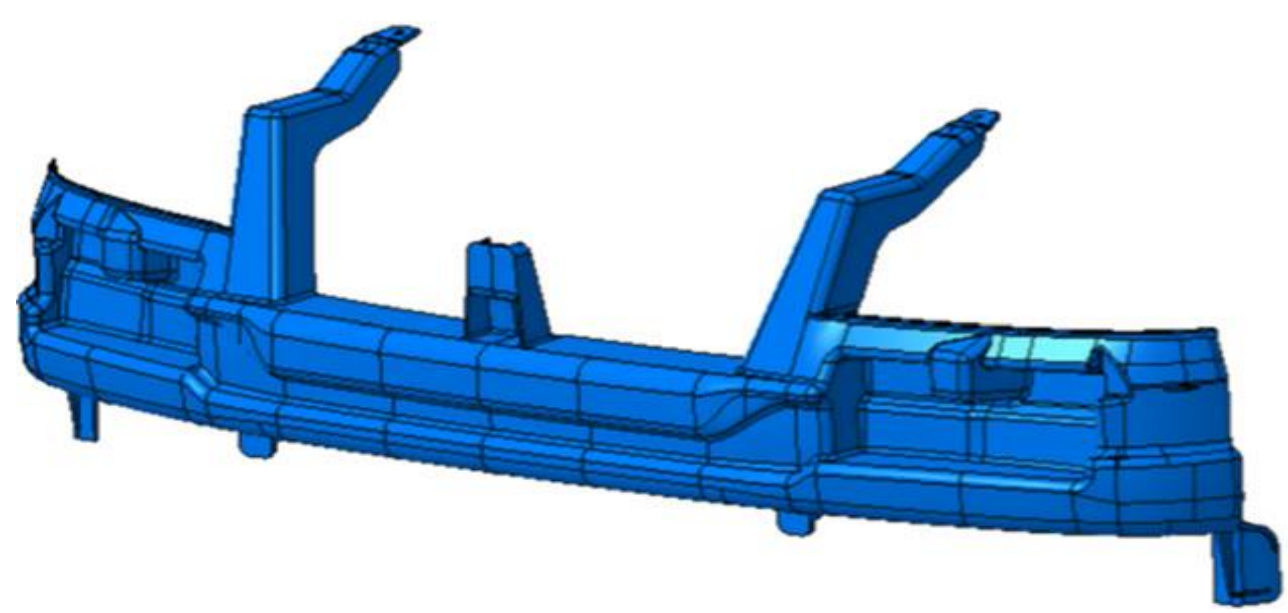

Fig. 3. GMT bumper beam of Samand [42].

the testing process for both sides is almost the same; the forward system should be stronger than the backward one for driver safety. On the other hand, the current trends in bumper design focus on aerodynamic efficiency where the designed curve should be embraced with the same style in other parts of the bumper system 
[22]. So, the conformable composite material solves this dilemma

by providing the required curvature and lowering the manufacturing cost, e.g., by multi-stage stamping of the metallic bumper beam, and decreases the beam weight [25].

Dissipation of energy by the bumper beam can be determined both by material and structural energy absorption [26]. The effective parameters in energy absorption of composite materials depend on type of fibre [27], matrix [28], fibre orientation [29], fabricating conditions [30], inter-laminar bond quality [31], and toughness [32]. The effective parameters of structural energy absorption are longitudinal curvature, cross-section profile [33], strengthening ribs [34], thickness [35], and the overall dimensions of the cross-section [36]. The energy absorption of material and structure was investigated by $[37,38]$. The crashworthiness of the vehicle and bumper system, which identifies the safety and performance of the vehicle in response to impact load, is a challenging issue. The enhanced performance of crashworthiness presents low damage to the vehicle and to occupants [39]. The impact energy in the bumper system can be dissipated reversibly (low impact) or irreversibly (crashworthiness) [40]. If the magnitude of the load does not exceed the elastic region "low-impact condition," then the structure returns to its previous position after releasing the load [4]. However, if the impact load goes beyond the elastic region "crashworthiness," then most of the collision load is absorbed by plastic deformation (irreversible energy absorption).

The bumper system should overcome both scenarios and sustain the intense load which results in large deformation, strain hardening, and various interactions between different deformation modes such as bending and stretching [41] (Fig. 3).

The proportion of energy reversibly absorbed by the bumper beam should be confined and the high kinetic energy should be preferably dissipated by plastic deformation. Otherwise, the collision energy maximizes the structural strain energy and release the same kinetic energy in return, which causes subsequent damage to the occupants or adjacent vehicles. Accordingly, the structural strain energy of the bumper beam should be optimized during the design process. Besides, ductility of material improves the plastic energy absorption. Within this context, plastic composites, polymer foams, and aluminium alloys are commonly used in the bumper systems when plastic energy damping and weight are critical design and performance criteria $[41,43]$.

\subsection{Bumper beam design parameters}

The stream of new materials, products, and process development has enforced a rethinking of the role of structural design 
and of the effective parameters for their improvement. The bumper beam can be improved by adjusting a number of effective parameters. The efficiency of the parameters can be identified by any of a number of methods such as design of experiments (DOE) [44], reliabilitybased design optimization (RBDO) [45], and design sensitivity analysis. However, the current study is not intended to identify the viability of the parameters. Variables such as thickness, bumper beam curvature, rib strength, and cross-section profile are some of the most important parameters which can improve the energy absorption of the bumper beam and sustain the desired deflection of the bumper system as defined in the product design specifications (PDSs). The optimal thickness of a bumper beam can construct a balance between the weight and strength of the structure in order to provide further effective energy absorption [46]. The nominal thickness of the bumper beam is $4 \mathrm{~mm}$. However, it is not completely constant in all beam parts. Surplus thickness of the polymer products has some manufacturing constraints. As an illustration, it increases the cooling time and makes warps in the flat surfaces and sink marks on the surface of the ribs' interface, which is not suitable in visible products

Strengthened ribs increases distortion resistance, rigidity, and structural stiffness through using little material in the slender walls [47] and providing the required impact severity [48]. Pattern, thickness, tip, and end fillet of the ribs should be designed according to load direction, impact position, material, and the manufacturing process available. Since the material thickness is high at the rib's contact area, it causes sink marks, but this is not much important a consideration, as a non-aesthetic part, for the bumper beam. It has been reported that the strengthened ribs increase the impact energy by $7 \%$ and decrease elongation by $19 \%[14,17,49]$. Zhang et al. [20] showed that the optimized reinforced ribs have higher-energy absorption performance than the empty and foamfilled beams.

Optimizing the cross-section of a bumper beam magnifies its strength, dimensional stability, and damping capability. It has significant effects on the energy damping rate and bending resistance compared with other parameters $[27,37]$. The right cross section can increase bumper beam strength and dimensional stability. Kim and Won [50] found that the section height is the most effective variable in torsional stiffness of the bumper beam. Additional strength permits more energy absorption with less consequent bumper beam distortion [51].

Frontal curvature increases the room between fixing points and top extremity beam curvature and increases the stability of the beam and the energy absorption. It enhances the beam 
stability and extends the required collision displacement. Besides

the aesthetic purposes, the curve facilitates additional load impact

distribution through the frontal beam and fixing points during

the energy damping process. When an impact load is applied

to the bumper, the beam initial curvature tends to restore its

original shape. So, some designers mounted a bar link between

the beam fixing points in order to strengthen the outward motion

and the energy absorption tendency [51,52]. The bumper

beam is an offset of the front bumper fascia that is intended

to provide a consistent level of protection across the vehicle

[53].

3. Material selection steps

Selecting a suitable material in bumper beam development is

crucial and bad selection may cause poor performance, frequent

maintenance or failure. Proper material selection for bumper beam

requires information about type of loading (axial, bending, torsion

or their combination), mode of loading (static, dynamic, fatigue,

impact), operating environment (temperature, humidity, chemical conditions), manufacturing process, cost (raw material, manufacturing,

assembly) [54].

Environmental constraints, economical demands, and performance

enhancement are main issues for material selection [55].

Material usually should be finalized in preliminary design stage,

while the material properties requirements are coupled with main

structural function [56]. The product function requirements usually

identify through product design specification (PDS) prior to

development process to guide the designer for precise selection

of design parameters and material selection. Then based on the

translated of product design specification, constraints, objectives,

geometry and process, which have interaction together the list of

material should be narrow down to the best candidate to comply

with the defined properties [57].

Physical, chemical, and mechanical properties along with manufacturing

and economic issues should be considered in selection

of a favorite material for a bumper beam [58]. Proper material

selection can be achieved by constructing a balance or compromise

between function, material, shape, and process [57]. The general

properties, processing, and performance of materials are considered

in the conceptual design phase and are refined into specific

requirements in the subsequent steps to ensure the performance

of the final product. Material selection of a bumper system usually

considers new environmental constraints, safety regulations, cost

reduction, reliability improvement, and performance enhancement.

Normally, the results of the failure analysis of previous products 
enable the designer to be more aware of material selection for the next product (Fig. 4)

There are two approaches for material selection of the bumper beam. Since manufacturing of the bumper beam is costly, the designers usually attempt to find the most consistent material for the available process that offers the desired properties. Otherwise, the material is selected initially and the optimized favorable manufacturing process is developed to meet the desired performance. Incorrect material selection and manufacturing method may lead to product failure, performance reduction, and cost increase.

Full text is available at :

http://ac.els-cdn.com/S0261306912002348/1-s2.0-S0261306912002348-main.pdf?_tid=dd3d6d26-a017-11e390f5-00000aacb361\&acdnat $=1393551190$ f7e37b1173bb865b3549510592a38779

http://www.sciencedirect.com/science/article/pii/S0261306912002348 